\title{
Observations of Low-Mass Protostars: Cold Envelopes and Hot Corinos
}

\author{
Cecilia Ceccarelli \\ Laboratoire d'Astrophysique de Grenoble, \\ 414 rue de la Piscine BP 53, 38041 Grenoble, France \\ email: Cecilia.Ceccarelli@obs.ujf-grenoble.fr
}

\begin{abstract}
Recent years have seen substantial progresses in our understanding of solar type protostellar structure, and particularly of the chemical structure of the protostellar envelopes. On the one hand, the cold outer regions keep intact the memory of the previous pre-collapse phase, when the dust is so cold and dense that almost all molecules freeze out onto the dust grain mantles. The gas-phase chemical composition undergoes dramatic changes, the most spectacular aspect of which is the huge increase of the molecular deuteration degree, which can reach 13 orders of magnitude with respect to the elemental $\mathrm{D} / \mathrm{H}$ ratio. On the other hand, in the innermost regions of the envelope - the so-called hot corinos - the grain mantles evaporate when the dust temperatures exceed $100 \mathrm{~K}$, injecting into the gas phase hydrogenated molecules, such as formaldehyde and methanol. Those molecules probably undergo chemical reactions that form more complex organic molecules, which have now also been observed in low-mass hot corinos. In this contribution, I review what we have and have not recently understood concerning both the cold envelopes and the hot corinos of solar-type protostars.
\end{abstract}

Keywords. astrochemistry — stars: formation

\section{Introduction}

Low-mass stars form from cold and dense condensations inside molecular clouds. The stages just before the collapse sets in are likely represented by the so-called pre-stellar cores (see Tafalla's and Shematovich's contributions in this volume). Once the collapse takes over, a protostar is formed: the central object - the future star - grows in mass by accreting material from the surrounding envelope, which, at the beginning, completely obscures it. This phase is likely represented by so-called Class 0 protostars: low-luminosity $\left(\leqslant 50 \mathrm{~L}_{\odot}\right)$ sources that emit most of their energy in the sub-millimeter wavelength range (André et al. 2000). The accretion of the central object - namely the infall motion of the envelope material - is accompanied by the spectacular phenomenon of outflow, which re-injects into the interstellar medium up to one-third of the infalling material. As time passes, the protostellar envelope is progressively dispersed: the protostar is believed to become a Class I source and then a pre-main-sequence star surrounded by a protoplanetary disk, which eventually may form comets, meteorites and planets (see Blake's and Kamp's contributions in this volume).

One of the major and more fascinating questions linked to the process of the formation of a star and its planetary system, especially if similar to our own solar system, is: What is the chemical budget acquired during the protostellar phase and inherited by the forming planets? Answering this question should shed some light on our own origins. This ultimate question splits into several smaller questions: What molecules are formed during the protostellar phase? Do molecules exist that are formed prevalently during the protostellar phase, and that are therefore a hallmark of this period? What is their fate? Do they condense onto the grain mantles during the proto-planetary phase? Are they 
incorporated into the planetesimals that eventually form comets, meteorites and planets? Are these pristine molecules released into the nascent planetary atmosphere during the early, intense comet bombardment? And what is the ultimate molecular complexity reached during star formation?

Since molecules formed during the protostellar phase might potentially be found at a later stage, even in planets, knowing the chemical composition of the protostellar envelope is therefore of paramount importance. This contribution deals with what we have learned in the last few years about the chemical composition of protostars in their first stage, the Class 0 phase. Although these sources are characterized by and even classified because of their cold appearance - the continuum emission of the cold $(\sim 30 \mathrm{~K})$ dust of the external regions of the envelope - it is now recognized that their envelopes have important temperature gradients $(\S 2)$. The cold outer shells hide inner regions where the temperature reaches the sublimation temperature of the grain mantles $(\sim 90-100 \mathrm{~K})$. From a chemical point of view, this divides the protostellar envelopes into two distinct regions: the outer envelope $(\S 3)$, where the chemistry is typical of cold and dense gas, and the inner envelope, where the chemistry is dominated by the mantle sublimation in warm gas. In $\S 4$, we will show that these inner regions share many of the characteristics of the hot cores discovered in high-mass protostars more than a decade ago (Kurtz et al. 2000). Even though they are not just a scaled version of the hot cores, the inner regions of low-mass protostars are now known as hot corinos (Ceccarelli 2004b).

\section{The Physical and Chemical Structure of Class 0 Protostars}

Class 0 sources are defined as low-luminosity sources, with a proven central object and centrally condensed envelope, whose luminosity in sub-millimeter to millimeter wavelengths, $L_{s m m}$, is an important fraction of the bolometric luminosity, $L_{b o l}: L_{s m m} / L_{b o l}$ $\geqslant 0.5 \%$ (André et al. 2000). This phenomenological classification is meant to correspond to a physical state: the mass of the inner hidden object is lower than the mass of the surrounding envelope. In the literature, other criteria have also been proposed and used, as, for example, the bolometric temperature (Chen et al. 1995). The goal of all the proposed criteria has been to identify the properties of protostars in the so-called "main accretion phase", i.e. the phase when the future star acquires the bulk of the mass. At present, fewer than 100 protostars have been counted and, to my knowledge, the most up-to-date database of Class 0 sources has been published by Froebrich (2005).

Evidently, the presence of a central object and a heating source implies the existence of a temperature gradient across the envelope. The first theoretical modeling of a spherical envelope undergoing collapse, and whose initial conditions are those of the singular isotherm sphere (SIS), as theorized by Shu and collaborators in the seventies (Shu 1977), was published by Ceccarelli et al. (1996). The model solves in a self-consistent way the gas thermal balance, the radiative transfer and the chemical composition across the envelope as functions of time. Successively, models with larger chemical networks have been developed (Rodgers \& Charnley 2003; Doty et al. 2004; Lee et al. 2004) and with inner photodissociation regions (PDRs) or X-ray-dominated regions (XDRs) (Stauber et al. 2004, 2005). Figure 1 shows the predicted physical structure of the envelope of IRAS 16293-2422, the prototype of Class 0 sources. The gas and dust temperature as well as the density profiles have been derived by modeling molecular line observations (Ceccarelli et al. 2000a,b).

The gas temperature tracks very closely the dust temperature, except in the very outer and inner regions. In the outer region $(\geqslant 400 \mathrm{AU})$, the CO lines are optically thin and, consequently, the CO line emission is particularly efficient in cooling the gas, which 

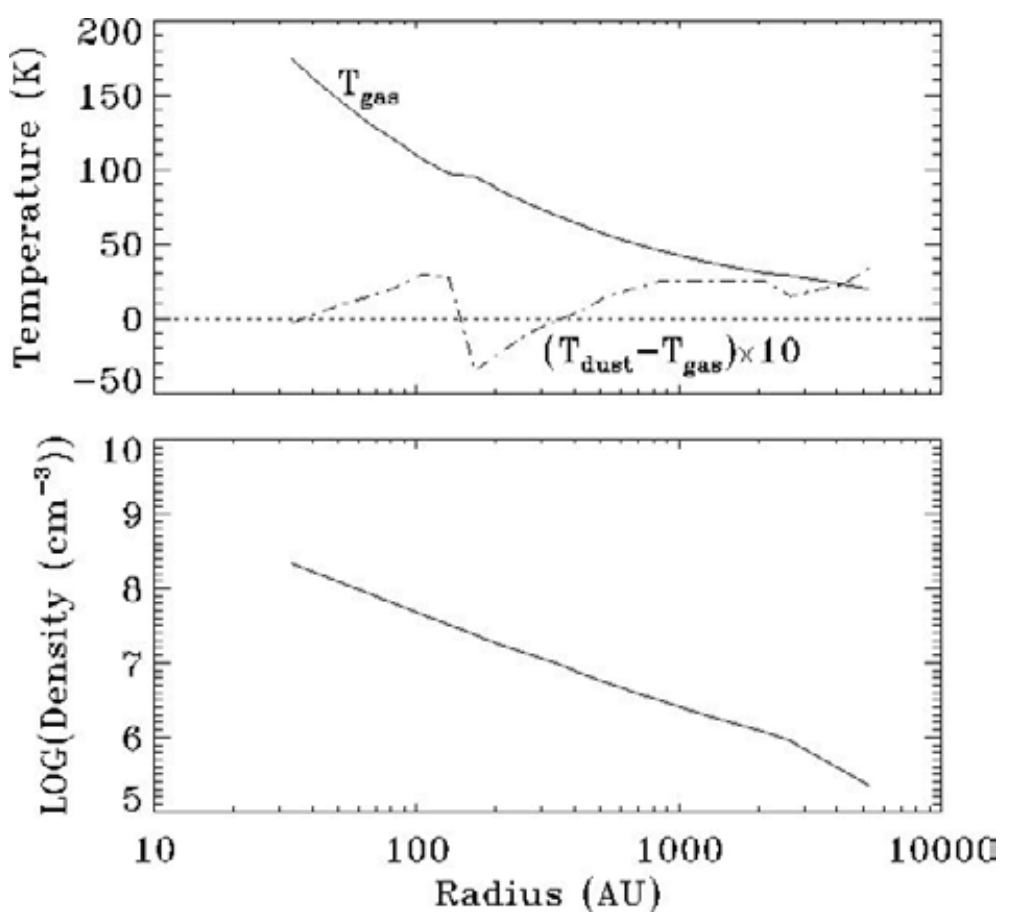

Figure 1. The temperature (upper panel) and density (lower panel) profile of the envelope surrounding the Class 0 protostar IRAS 16293-2422 (Figure from Ceccarelli et al. 2000a).

becomes cooler than the dust. On the contrary, in the inner regions between about 150 and $400 \mathrm{AU}$, the gas is warmer than the dust, because the CO and O lines (the major gas coolants in that region) are optically thick and, therefore, not very efficient in cooling the gas. At about $150 \mathrm{AU}$, the grain mantles sublimate injecting into the gas phase a large quantity of water molecules. However, the water molecules become more a heating agent than a cooling one, because $\mathrm{H}_{2} \mathrm{O}$ molecules are pumped radiatively by the NIR photons of the warm dust and de-excited by collisions, giving rise to a net heating rate. A similar analysis has been carried out for another Class 0 source, NGC 1333 IRAS 4 (Maret et al. 2002). Basically, the same behavior observed in IRAS 16293-2422 applies to this protostar too.

Another approach used in the literature is that of deriving the dust temperature and density of the protostellar envelopes by modeling the continuum emission, specifically the spectral energy distribution (SED) and the spatial distribution (maps) simultaneously (e.g., Jorgensen et al. 2002). In this case the dust density is assumed to follow a power law, and the gas temperature to be identical to the dust temperature. The two methods give approximatively the same results, which likely just means that the approximations used are valid when considering the uncertainties associated with the observational data. Moreover, this also means that one can rely, to a first approximation, on the predictions obtained by these models.

In the context of this review, the important result of these models is the prediction of regions with dust temperatures larger than the sublimation temperature of the grain mantles $(\sim 100 \mathrm{~K})$. This prediction has some very immediate consequences: in this region grain mantles evaporate, and, therefore, the abundance of the mantle components should increase with respect to the abundance in the cold $\left(T_{\text {dust }} \leqslant 100 \mathrm{~K}\right)$ envelope. Water is the major component of the ice mantles, but unfortunately water lines are not observable 


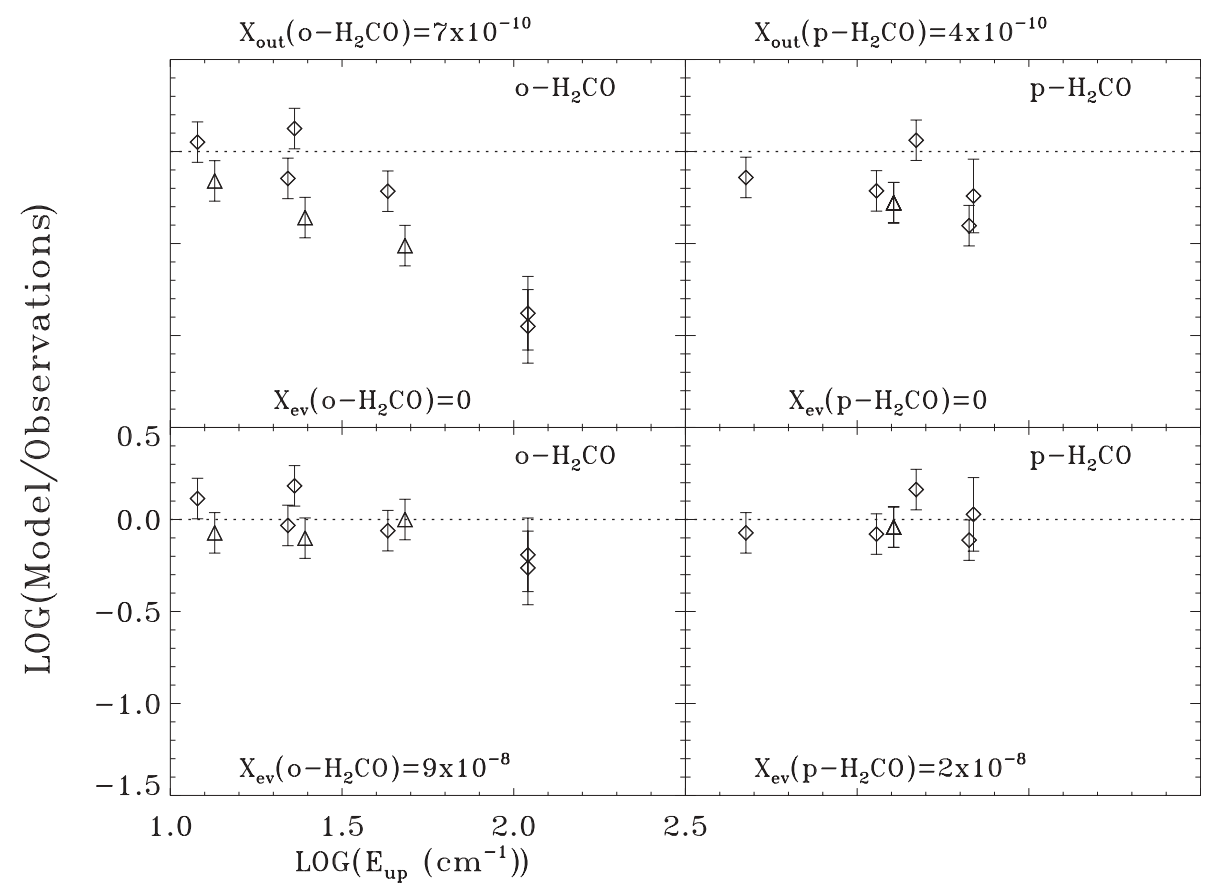

Figure 2. Ratio between the observed and predicted line fluxes as a function of the upper level energy for a model with the parameters described in the text (bottom panels) and for a model with no evaporation in the inner region (top panels). The diamonds represent the $\mathrm{H}_{2}^{12} \mathrm{CO}$ lines while the triangles the $\mathrm{H}_{2}^{13} \mathrm{CO}$ lines. Note that the logarithm of the upper level energies of the $\mathrm{H}_{2}^{13} \mathrm{CO}$ lines has been shifted by 0.05 to make the points distinguishable in the plot (Figure from Ceccarelli et al. 2000b).

with ground-based telescopes. The spectrometer LWS aboard ISO provided observations of water lines towards a few Class 0 sources (Ceccarelli et al. 1999), but with a relatively poor spatial and spectral resolution, so that the interpretation of the data is not unique. Nonetheless, if the water line spectra are interpreted as emitted in the envelope, allowing a jump in the water abundance in the $T_{d u s t} \geqslant 100 \mathrm{~K}$ region, the agreement among the model predictions and the observed line fluxes is rather good. The analysis has been carried out for two cases: IRAS 16293-2422 and NGC 1333 IRAS 4 (Ceccarelli et al. 2000a; Maret et al. 2002). In both cases, the observed data are reproduced if the water abundance is $\sim 3 \times 10^{-7}$ in the outer envelope, and jumps by about a factor of 10 in the inner region. Formaldehyde is also an important grain-mantle component and this molecule has the advantage that its transitions lie in the millimeter to sub-millimeter wavelength range and are, therefore, observable with ground-based telescopes. These observations have a much better spatial and spectral resolution than ISO-LWS and provide stronger constraints on the models. Figure 2 shows the ratio between the theoretical predictions and the observed line fluxes towards IRAS 16293-2422 for two cases: with a $\mathrm{H}_{2} \mathrm{CO}$ abundance constant across the envelope, and with the $\mathrm{H}_{2} \mathrm{CO}$ abundance profile following a jump function (Ceccarelli et al. 2000b). The constant abundance model fails to reproduce the observed $\mathrm{H}_{2} \mathrm{CO}$ spectrum, because the transitions with high $(\geqslant 70 \mathrm{~K})$ upper energies are underestimated by one order of magnitude. The abundance jump model instead reproduces the observations quite well. The presence of a jump in the abundance of formaldehyde lends strong support to the hypothesis of the mantle ice sublimation.

A similar analysis, in terms of abundance jump models, has been carried out with several other molecules in IRAS 16293-2422 (Schöier et al. 2002). Molecules, like $\mathrm{HC}_{3} \mathrm{~N}$, 

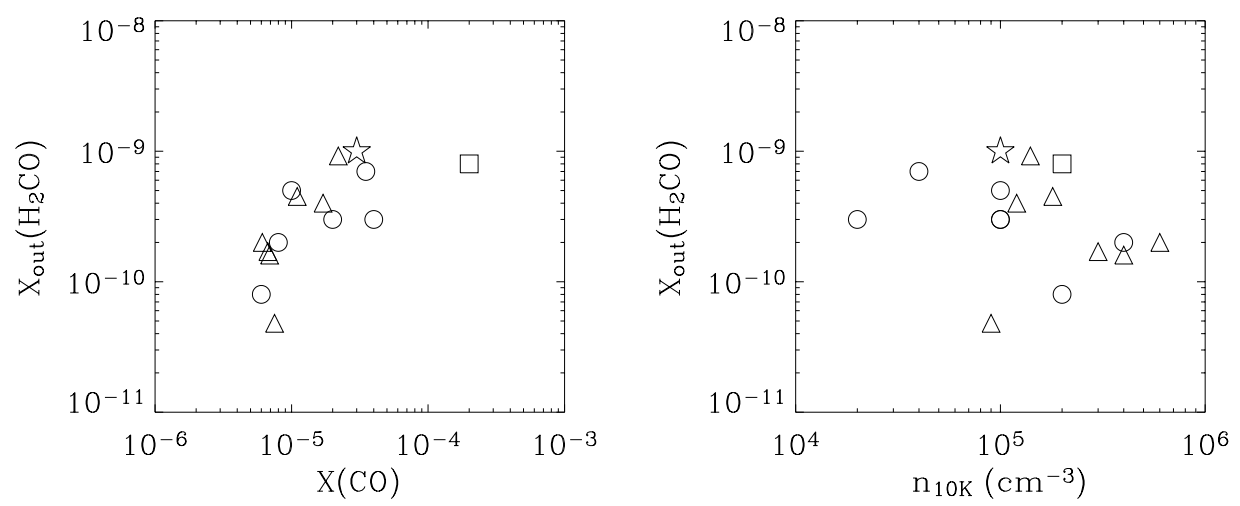

Figure 3. The abundances of $\mathrm{H}_{2} \mathrm{CO}$ and $\mathrm{CO}$ in the outer envelopes of Class 0 sources and in pre-stellar cores (left panel) and the $\mathrm{H}_{2} \mathrm{CO}$ abundance versus the density where $T_{\text {dust }}=10 \mathrm{~K}$ (right panel). Circles represent Class 0 sources, the star represents IRAS 16293-2422, the square represents VLA1623 - the only targeted source where no $\mathrm{H}_{2} \mathrm{CO}$ jump could be detected - and the triangles represent the pre-stellar cores of the Bacmann et al. (2002) sample (Figure from Maret et al. 2004).

$\mathrm{CH}_{3} \mathrm{CN}, \mathrm{CH}_{3} \mathrm{OH}$, SO and $\mathrm{SO}_{2}$, which are thought to be frozen out onto the grain mantles, display jumps in their abundance by more than one order of magnitude. Also, this work confirms the basic prediction of a region, where the grain mantles sublimate.

All these studies refer to the case of IRAS 16293-2422. The question arises whether IRAS 16293-2422 is representative of Class 0 sources, or it is a peculiar object in the astronomical zoo. A survey of the $\mathrm{H}_{2} \mathrm{CO}$ line emission towards a bit less than a dozen Class 0 sources has shown that all the targeted sources, except VLA16293, have a region where the formaldehyde abundance jumps by more than one order of magnitude (Maret et al. 2004). Several of these sources also show jumps in the methanol abundance (Maret et al. 2005). The sizes of the jump regions range from 10 to $150 \mathrm{AU}$ (see Table 1), and are, therefore, comparable to the size of the solar system. Both the estimates of the abundances and the warm region sizes suffer a relatively large uncertainty, for they are derived by the multi-frequency analysis of single-dish measurements, which encompass regions between 1000 and 5000 AU. Finally, an additional uncertainty is also connected to the details of the model used to interpret the data (Jorgensen et al. 2005b). In the following, I will review the characteristics of the outer and inner regions respectively, giving further arguments in favor of this structure.

\section{The Cold Outer Envelopes}

The most outstanding characteristic of the outer envelopes is that, from the chemical point of view, they do not seem to be touched by the collapse. They keep intact the memory of the pre-collapse phase, for they are chemically identical to pre-stellar cores. This similarity is based on two key properties of the outer envelopes and pre-stellar cores: (i) the large depletion of CO and the heavy-element-bearing molecules; and (ii) the strong enhancement of molecular deuteration.

Figure 3 shows the abundance of $\mathrm{H}_{2} \mathrm{CO}$ and $\mathrm{CO}$ in the outer envelopes of Class 0 sources (Maret et al. 2004) and in a sample of pre-stellar cores (Bacmann et al. 2002). 


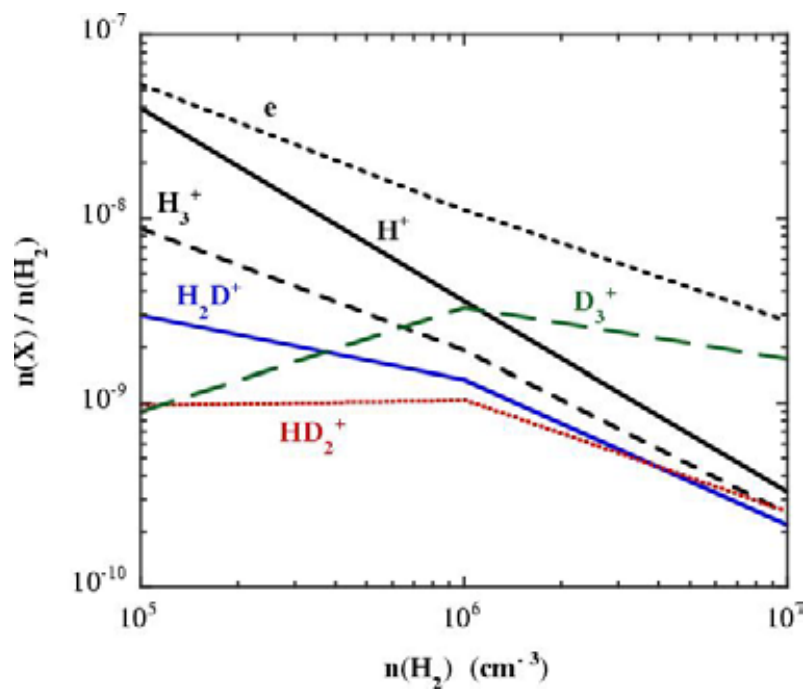

Figure 4. Abundances of major ions and electrons in the standard model (dust radius = $0.1 \mu \mathrm{m}, \mathrm{T}=10 \mathrm{~K}, \zeta=3 \times 10^{-17} \mathrm{~s}^{-1}$ ) as functions of the density of molecular hydrogen (Figure from Walmsley et al. 2004).

First, $\mathrm{CO}$ and $\mathrm{H}_{2} \mathrm{CO}$ are highly depleted, up to a factor almost 100 . This is due to the condensation of these molecules on the grain mantles. Second, the two samples are practically indistinguishable on this plot, suggesting that indeed the pre-stellar cores are likely the precursors of Class 0 sources. The other amazing aspect of Figure 3 is the tight linear correlation between the $\mathrm{CO}$ and $\mathrm{H}_{2} \mathrm{CO}$ abundances. In principle, the binding energies of $\mathrm{CO}$ and $\mathrm{H}_{2} \mathrm{CO}$ are different, and $\mathrm{H}_{2} \mathrm{CO}$ should freeze-out onto the grain mantles at lower densities than $\mathrm{CO}$. The observed correlation suggests a common chemical origin for the two species.

The second key aspect pre-stellar cores and Class 0 outer envelopes share is the strongly enhanced molecular deuteration. When considering the processes at the basis of molecular deuteration, this is not surprising, but actually is a tautology with respect to the first aspect. It is in fact the diminishing of the $\mathrm{CO}$ abundance in the gas phase that causes the extreme molecular deuteration observed in both classes of objects. In fact, the deuterium atoms are transmitted to the molecules by proton exchange with $\mathrm{H}_{2} \mathrm{D}^{+}$, which is formed by the reaction of $\mathrm{H}_{3}^{+}$with $\mathrm{HD}$. Under standard conditions, $\mathrm{H}_{3}^{+}$is destroyed by the reaction with $\mathrm{CO}$ (the most abundant molecule after $\mathrm{H}_{2}$ ) and, to a much smaller extent, by electron recombination and reaction with HD. However, in CO-depleted and low-ionization gas, the major destruction route of $\mathrm{H}_{3}^{+}$becomes the reaction with HD which forms $\mathrm{H}_{2} \mathrm{D}^{+}$. As a consequence, $\mathrm{H}_{2} \mathrm{D}^{+}$can become even more abundant than $\mathrm{H}_{3}^{+}$. Actually, in extreme CO-depletion conditions, $\mathrm{H}_{2} \mathrm{D}^{+} / \mathrm{H}_{3}^{+}$reaches the value of 2 , and the most abundant ion can become $\mathrm{D}_{3}^{+}$, the final product of the chain that forms $\mathrm{H}_{2} \mathrm{D}^{+}$, $\mathrm{HD}_{2}^{+}$and $\mathrm{D}_{3}^{+}$, as shown in Figure 4 (Roberts et al. 2003; Flower et al. 2004; Ceccarelli \& Dominik 2005).

The new classes of models for molecular deuteration mentioned above were triggered by the numerous observations of dramatically enhanced $\mathrm{D} / \mathrm{H}$ ratios, proved by the detection of several doubly and triply deuterated molecules. When considering that the cosmic elemental abundance of deuterium is $\sim 10^{-5}$ times the hydrogen abundance, the adjective "dramatic" is barely an exaggeration. Although doubly deuterated formaldehyde had 
been detected fifteen years ago in Orion by Turner (1990), the measured abundance $\left(\sim 0.2 \%\right.$ of $\left.\mathrm{H}_{2} \mathrm{CO}\right)$ did not seem to draw particular attention (probably because Orion has always been considered a peculiar region). The discovery that $\mathrm{D}_{2} \mathrm{CO}$ has an abundance about $5 \%$ that of $\mathrm{H}_{2} \mathrm{CO}$ in a low-mass protostar, IRAS 16293-2422 (Ceccarelli et al. 1998), on the contrary, started a flurry of activity in this field, and opened the quest for multiply deuterated molecules. In a few years, the following deuterated species have been detected (see Ceccarelli 2004a for a recent review of the subject): doubly and triply deuterated ammonia (Roueff et al. 2000; Loinard et al. 2001; Lis et al. 2002; van der Tak et al. 2002), doubly deuterated hydrogen sulfide (Vastel et al. 2003), and doubly and triply deuterated methanol (Parise et al. 2002, 2004). In addition, the following detailed studies of doubly deuterated formaldehyde have been carried out:

- Towards IRAS 16293-2422, to characterize and better constrain the exact physical conditions of the deuterated gas (Loinard et al. 2000);

- Towards IRAS 16293-2422 to measure the deuteration ratio across the envelope, with results larger at $\sim 2000 \mathrm{AU}$ from the center, i.e. in the outer envelope (Ceccarelli et al. 2001);

- In a sample of protostars, to prove that IRAS 16293-2422 is not a peculiarity but representative of Class 0 sources with respect to the observed degree of formaldehyde deuteration (Loinard et al. 2002);

- In a sample of pre-stellar cores, to prove that the molecular deuteration observed in the Class 0 sources sets in during the pre-collapse phase (Bacmann et al. 2003).

All these observational studies helped to elucidate the mechanisms underlying the observed molecular deuteration (see Roberts' contribution in this volume). There exist two main routes, depending on the molecule: in the gas phase (Roberts \& Millar 2000) or on the grain surfaces (Tielens 1983). Likely, ammonia is a gas-phase product (Roueff et al. 2000), whereas methanol and formaldehyde are grain-surface products (Ceccarelli et al. 2001; Parise et al. 2002). Whatever the mechanism, at the heart of the two routes is the enhancement of the deuteration of $\mathrm{H}_{3}^{+}$. In the first case, gas-phase chemistry, the deuterated forms of $\mathrm{H}_{3}^{+}$directly transmit the deuteration to the other molecules (see above). In the second case, grain-surface chemistry, the deuteration is mostly due to the accreting gas at the moment of mantle formation, which, in turns, depends on the deuterated forms of $\mathrm{H}_{3}^{+}$. In this respect, a key detection was that of $\mathrm{H}_{2} \mathrm{D}^{+}$towards the pre-stellar core L 1544 (Caselli et al. 2003). This key ion had been searched for decades without success toward massive star forming regions, and it was finally detected with an abundance comparable to that of $\mathrm{H}_{3}^{+}$in this little and cold condensation. This observation was likely the last step that led to the new class of chemical models described at the beginning of this paragraph. Finally, the detection of $\mathrm{HD}_{2}^{+}$was one important confirmation of the proposed new schemes (Vastel et al. 2004).

Although our comprehension of the mechanisms leading to molecular deuteration in the interstellar medium has undoubtedly improved in these last few years, there still remain a number of unresolved questions. First, what is the relative role of gas-phase versus grain-surface chemistry? It is not completely clear yet what molecules are formed in one or the other way, and to what extent. This comes along with the fact that the models still have difficulties in reproducing all the details of the observations. Figure 5 illustrates an example linked to formaldehyde and methanol formation. Both molecules are thought to be formed on grain surfaces via successive hydrogenation of CO (Tielens \& Hagen 1982; Charnley et al. 1997). Based on this theory, the deuteration ratios in the two molecules should be approximatively similar, because both molecules form almost simultaneously when $\mathrm{CO}$ condenses out onto the grain mantles. However, observations of the various forms of the two molecules do not fully agree with this picture, as shown 


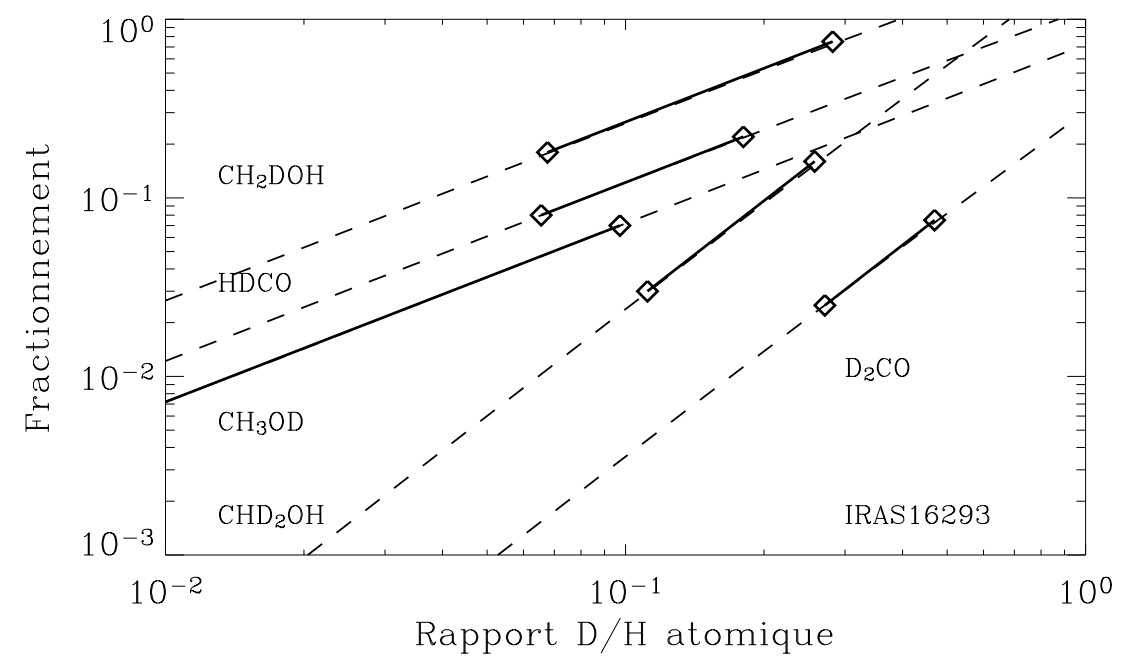

Figure 5. Comparison between the observed fractionations towards IRAS 16293-2422 (solid lines) and the predictions of the grain model (dashed lines) by Stantcheva \& Herbst (2003). The data are from Parise et al. (2002) and Parise et al. (2004) and the Figure has been kindly provided by B. Parise.

in Figure 5. It refers to the case of IRAS 16293-2422, but a survey of several Class 0 sources confirms that similar plots can be drawn for the other surveyed sources (Parise et al., in preparation).

There is a slight discrepancy between the value of the required $\mathrm{D} / \mathrm{H}$ in accreting atomic gas, when considering the various deuterated species. $\mathrm{D}_{2} \mathrm{CO}$ requires systematically larger atomic $\mathrm{D} / \mathrm{H}$ ratios than $\mathrm{HDCO}$ and any other form of deuterated methanol. The $\mathrm{CH}_{3} \mathrm{OD} / \mathrm{CH}_{3} \mathrm{OH}$ ratio is systematically much lower than that of the other forms of methanol. At this point it is still not completely clear whether this is because of gasphase reactions taking place after these molecules are injected into the gas phase after the mantle sublimation (Parise et al. 2002; Osamura et al. 2004).

Another puzzle is represented by deuterated water. Searches for solid HDO have been negative so far, giving some stringent upper limits to the water deuteration. In particular, the value of $\leqslant 2 \%$ (Dartois et al. 2003; Parise et al. 2003) is considerably less than the deuteration observed in the other molecules. However, observations of solid $\mathrm{H}_{2} \mathrm{O}$ and HDO may be "contaminated" by the contribution of the molecular cloud in front of the observed protostar (see for example the discussion in Boogert et al. 2002). A more stringent constraint is set by observations of gas-phase HDO in the outer envelope and hot corino, where the icy mantles sublimate. Observations towards IRAS 16293-2422 set some stringent constraint to the deuteration of water: in the sublimated ices it is $\sim 3 \%$, whereas in the cold envelope it is $\leqslant 0.2 \%$ (Parise et al. 2005). Even considering a water abundance in the ices of $10^{-4}$, the measured $\mathrm{HDO} / \mathrm{H}_{2}$ of $3 \%$ is still low with respect to the available elemental deuterium, by about a factor 5 . Therefore, water deuteration seems to follow a different route than formaldehyde, methanol, and the other molecules. 
Table 1. Properties of the inner envelopes of Class 0 sources.

\begin{tabular}{lcccccr}
\hline Source & Radius & Density & \multicolumn{2}{c}{$\mathrm{H}_{2} \mathrm{CO}$} & \multicolumn{2}{c}{$\mathrm{CH}_{3} \mathrm{OH}$} \\
& $(\mathrm{AU})$ & $\left(\mathrm{cm}^{-3}\right)$ & Outer & Inner & Outer & \multicolumn{1}{c}{ Inner } \\
\hline NGC 1333-IRAS 4A & 53 & $2 \times 10^{9}$ & $2 \times 10^{-10}$ & $2 \times 10^{-8}$ & $7 \times 10^{-10}$ & $<1 \times 10^{-8}$ \\
NGC 1333-IRAS 4B & 27 & $2 \times 10^{8}$ & $5 \times 10^{-10}$ & $3 \times 10^{-6}$ & $2 \times 10^{-9}$ & $7 \times 10^{-7}$ \\
NGC 1333-IRAS 2 & 47 & $3 \times 10^{8}$ & $3 \times 10^{-10}$ & $2 \times 10^{-7}$ & $1 \times 10^{-9}$ & $3 \times 10^{-7}$ \\
L 1448-MM & 20 & $2 \times 10^{8}$ & $7 \times 10^{-10}$ & $6 \times 10^{-7}$ & $2 \times 10^{-9}$ & $5 \times 10^{-7}$ \\
L 1448-N & 20 & $1 \times 10^{8}$ & $3 \times 10^{-10}$ & $1 \times 10^{-6}$ & $7 \times 10^{-10}$ & $<4 \times 10^{-7}$ \\
L 1157-MM & 40 & $8 \times 10^{8}$ & $8 \times 10^{-11}$ & $1 \times 10^{-8}$ & $3 \times 10^{-10}$ & $<3 \times 10^{-8}$ \\
IRAS 16293-2422 & $2 \times 10^{8}$ & 133 & $1 \times 10^{-9}$ & $1 \times 10^{-7}$ & $1 \times 10^{-9}$ & $1 \times 10^{-7}$ \\
\hline
\end{tabular}

Notes: The second column reports the radius, at which the dust temperature exceeds the grainmantle sublimation temperature $(\sim 100 \mathrm{~K})$. The following columns report the formaldehyde and methanol abundances in the outer and inner envelope respectively (from Maret et al. 2004, 2005).

\section{The Hot Corinos}

Table 1 summarizes some important known properties of the inner regions, the hot corinos.

Hot corinos have sizes comparable to the sizes of the solar system, with radii smaller than about 50 AU, with the notable exception of IRAS 16293-2422, which, on this very particular aspect is not representative of the other Class 0 sources. The density is relatively large, larger than $10^{8} \mathrm{~cm}^{-3}$ in all studied hot corinos. The abundances of formaldehyde and methanol jump by more than one order of magnitude with respect to the abundances in the outer envelopes, supporting the theory that grain mantles sublime in these regions.

A key question, and one relevant for the chemical structure of the Class 0 sources, is what molecules are found in the hot corinos and, in particular, whether organic complex molecules are formed, in analogy with massive hot cores. More generally, are hot corinos scaled versions of hot cores, or does a peculiar chemistry take place? And how reliable are the hot corino sizes derived from the analysis of the single-dish data?

Once again, the benchmark for these studies has been IRAS 16293-2422. In the hot corino of this source, complex organic molecules were detected for the first time (Cazaux et al. 2003), and this hot corino was also the first to be imaged (Kuan et al. 2004; Bottinelli et al. 2004b).

Figure 6 shows the extremely rich spectrum of IRAS $16293-2422$ at $98 \mathrm{GHz}$, and the lines from identified complex molecules. In practice, all the complex molecules typical of massive hot cores searched for were detected, with abundances similar to, if not higher than, those measured in the hot cores (Cazaux et al. 2003), including $\mathrm{O}$ and N-bearing molecules such as formic acid, $\mathrm{HCOOH}$, acetaldehyde, $\mathrm{CH}_{3} \mathrm{CHO}$, methyl formate, $\mathrm{CH}_{3} \mathrm{OCHO}$, dimethyl ether, $\mathrm{CH}_{3} \mathrm{OCH}_{3}$, acetic acid, $\mathrm{CH}_{3} \mathrm{COOH}$, methyl cyanide, $\mathrm{CH}_{3} \mathrm{CN}$, ethyl cyanide, $\mathrm{C}_{2} \mathrm{H}_{5} \mathrm{CN}$ and propyne, $\mathrm{CH}_{3} \mathrm{CCH}$. Two years after the first detection in IRAS 16293-2422, complex organic molecules have also been detected towards the other Class 0 sources NGC 1333 IRAS 4A and B, and NGC 1333 IRAS 2 (Bottinelli et al. 2004a; Jorgensen et al. 2005a; Bottinelli et al. 2005b).

The number of hot corinos, where complex organic molecules have been detected is evidently much too small to draw any conclusions based on statistical considerations (see Table 2). However, a first conclusion is that complex organic molecules seem to be a rather common product in hot corinos. In this aspect, IRAS 16293-2422 does not seem peculiar, and may be representative of the Class 0 sources. However, there is a relatively 


\section{Rest Frequency $(\mathrm{GHz})$}

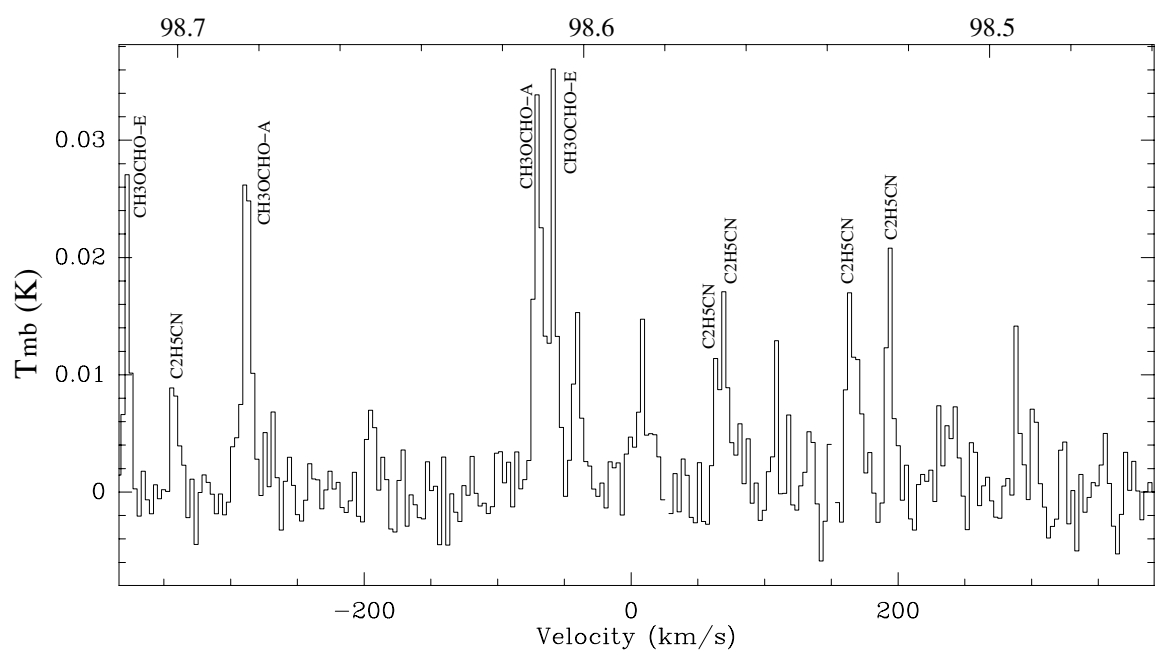

Figure 6. The spectrum at $98 \mathrm{GHz}$ of IRAS 16293-2422 (Cazaux et al. 2003).

Table 2. Abundances of the complex organic molecules observed in Class 0 sources. ${ }^{\dagger}$

\begin{tabular}{|c|c|c|c|c|}
\hline Molecule & IRAS 16293 & IRAS $4 \mathrm{~A}$ & IRAS 4B & IRAS $2 \mathrm{~A}$ \\
\hline $\mathrm{H}_{2} \mathrm{CO}$ & $1 \times 10^{-7}$ & $2 \times 10^{-8}$ & $3 \times 10^{-6}$ & $2 \times 10^{-7}$ \\
\hline $\mathrm{CH}_{3} \mathrm{OH}$ & $1 \times 10^{-7}$ & $<1 \times 10^{-8}$ & $3 \times 10^{-6}$ & $2 \times 10^{-7}$ \\
\hline $\mathrm{HCOOCH}_{3}-\mathrm{A}$ & $1.7 \times 10^{-7}$ & $3.4 \times 10^{-8}$ & $1.1 \times 10^{-6}$ & $<6.7 \times 10^{-7}$ \\
\hline $\mathrm{HCOOH}$ & $6.2 \times 10^{-8}$ & $4.6 \times 10^{-9}$ & $<1.0 \times 10^{-6}$ & $<1.2 \times 10^{-7}$ \\
\hline $\mathrm{CH}_{3} \mathrm{OCH}_{3}$ & $2.4 \times 10^{-7}$ & $<2.8 \times 10^{-8}$ & $<1.2 \times 10^{-6}$ & $3.0 \times 10^{-8}$ \\
\hline $\mathrm{CH}_{3} \mathrm{CN}$ & $1.0 \times 10^{-8}$ & $1.6 \times 10^{-9}$ & $9.5 \times 10^{-8}$ & $8.7 \times 10^{-9}$ \\
\hline $\mathrm{C}_{2} \mathrm{H}_{5} \mathrm{CN}$ & $1.2 \times 10^{-8}$ & $<1.2 \times 10^{-9}$ & $<7.5 \times 10^{-7}$ & $<1.0 \times 10^{-7}$ \\
\hline
\end{tabular}

${ }^{\dagger}$ Bottinelli et al. (2005b)

large spread in the derived abundances of the detected organic complex molecules. At this point it is not clear what causes this spread, whether it is the age of the hot corino, the mantle composition, or something else.

So far, results based on single-dish observations have been discussed. As previously remarked, these observations encompass regions of at least $1000 \mathrm{AU}$, whereas the predicted sizes of the hot corinos do not exceed 150 AU (Table 1). It is therefore more than legitimate to verify these predictions with high-spatial resolution, interferometric observations.

The first images of a hot corino have been obtained in the direction of IRAS 162932422 with the interferometers SMA (Kuan et al. 2004; Chandler et al. 2005) and PdB (Bottinelli et al. 2004b). Figure 7 shows the images obtained at $\mathrm{PdB}$ in the $1.3 \mathrm{~mm}$ continuum and in one methyl format line (Bottinelli et al. 2004b). Here are a few striking remarks:

- The line emission is concentrated in two spots, centered on the two objects forming the proto-binary system. The whole line flux detected with the single dish is recovered in the two spots, which means that all the single-dish emission originates in those two spots. 

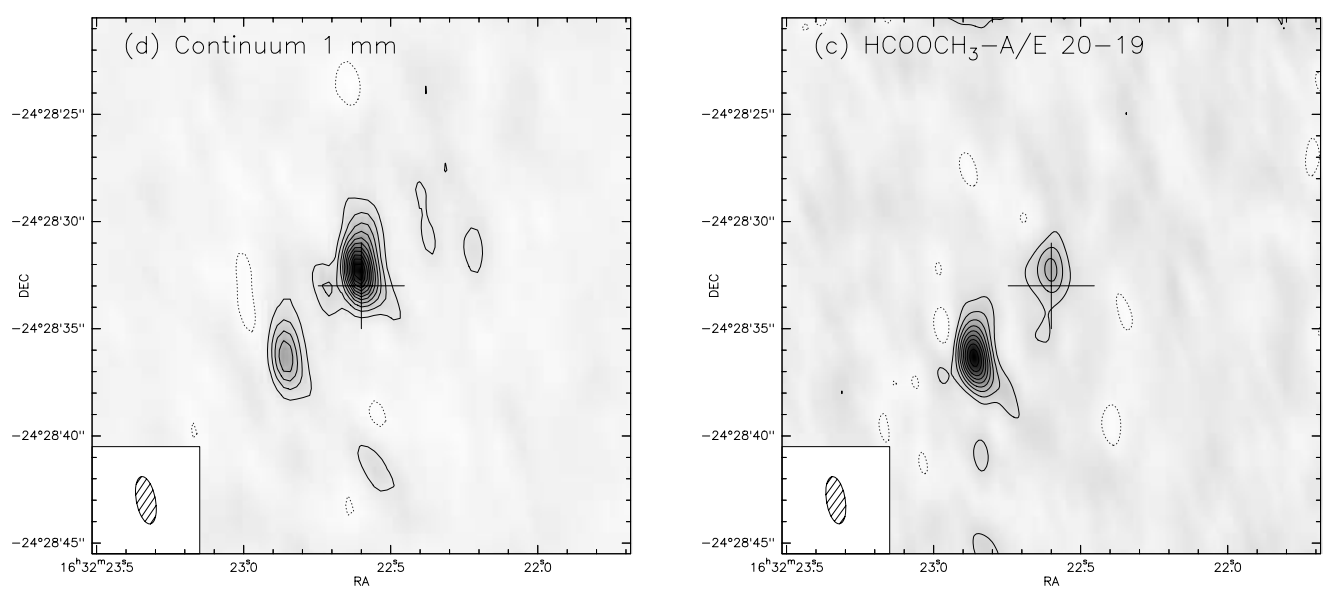

Figure 7. PdB images of IRAS 16293-2422 (Bottinelli et al. 2004b): the $1.3 \mathrm{~mm}$ continuum (left panel) and a line of methyl formate (right panel).

- No signs of outflowing material are associated with the methyl formate emission: the emission is compact and the line profile is relatively narrow.

- The brightest - and resolved - spot has a radius of $\sim 150 \mathrm{AU}$, exceedingly close to the "old" predictions, based on the low-resolution water-line spectrum by ISO-LWS (Ceccarelli et al. 2000a).

- The two objects of the proto-binary system forming IRAS 16293-2422 do not appear to have the same characteristics. The north source ( $\mathrm{B}$ in the literature) is bright in the $1.3 \mathrm{~mm}$ continuum, but barely detected in the methyl format line. On the contrary, the south source shines in the molecular line and is barely detected in the continuum.

PdB images of NGC 1333 IRAS 4 have recently been obtained by Bottinelli et al. (2005a). As in the case of IRAS 16293-2422, IRAS 4A is a binary system, and the observed ethyl cyanide line emission is concentrated in two spots, centered on the two proto-binary system objects. Again, the two objects do not have the same appearance, with the strength in the continuum and line emission once again reversed.

It is to be emphasized, however, that in both cases, the fact that the two binary objects have a different appearance in the line emission does not necessarily imply that the abundance in the two hot corinos is different. In fact, the weakest hot corino in both binary systems is unresolved and the line likely optically thick (see the discussion in Bottinelli et al. 2004b). Evidently, high-resolution observations of organic molecules are a valuable tool to study the chemistry of the hot corinos, and how it depends on the physical conditions, rather than the mantle composition and age, which presumably are the same for the two objects of the binary system. In this respect, it is worth mentioning the potential of the sulfur-bearing molecules in estimating the age of the hot corinos. For many years, S-bearing molecules have been thought to be potential "chemical clocks" (Charnley 1997; Hatchell et al. 1999; Wakelam et al. 2004a). Recently, Wakelam et al. (2004a) have shown that although it is a very delicate exercise, which requires sophisticated modeling, measurements of the $\mathrm{SO}, \mathrm{SO}_{2}, \mathrm{H}_{2} \mathrm{~S}$ and $\mathrm{CS}$ can indeed give useful constraints on the hot corino age (see also Wakelam et al. 2004b, 2005). The method applied to IRAS 16293-2422 gives an estimate of the hot corino age of about $3000 \mathrm{yr}$ (Wakelam et al. 2004a). Note that this is the age of the hot corino and not the age of the protostar, though. In other words, this is the time since the ice mantles sublimated in the $150 \mathrm{AU}$ radius region, which depends on the density and luminosity of the source. 
Therefore, this time does not account for the time required to reach that luminosity and density.

\section{Conclusions}

In this contribution, new progress reached in these last years about the chemical composition of Class 0 sources has been reported. It has been shown that the envelopes surrounding the accreting future stars have a relatively simple chemical structure. Molecules are formed in two distinct regions:

(i) The outer envelope, defined by where the dust temperature is lower than the sublimation temperature of the grain mantles. From a chemical point of view, the outer envelopes are practically identical to pre-stellar cores, sharing with them a large molecular depletion and an extreme molecular deuteration.

(ii) The hot corino, where the chemistry is dominated by sublimation of the grain mantles. These regions are dense $\left(\sim 10^{8} \mathrm{~cm}^{-3}\right)$, warm $(\geqslant 100 \mathrm{~K})$ and compact $(\leqslant 150$ $\mathrm{AU}$ in radius). Their major characteristic is an enrichment in complex organic molecules.

While several studies have appeared in the literature on cold envelope chemistry, much less is known about the hot corinos, and their nature is still debated (as is evident by the questions asked during the presentation of this review). I expect that substantial progress will be obtained in the incoming years, thanks to the exploitation of available instruments, the advent of new instruments like ALMA, as well as the development of new chemical models for the hot corinos.

\section{Acknowledgements}

I would like to acknowledge and thank the enthusiastic support of the WAGOS group, which did much of the work presented in this review, specifically A. Bacmann, S. Bottinelli, A. Castets, E. Caux, S. Cazaux, B. Lefloch, S. Maret, B. Parise, L. Pagani, X. Tielens, C. Vastel and V. Wakelam. I also acknowledge the financial support of the French National Program entitled "Physique et Chimique du Milieu Interstellaire".

\section{References}

André, P., Ward-Thompson, D., \& Barsony, M. 2000, Protostars and Planets IV, 59

Bacmann, A., Lefloch, B., Ceccarelli, C., Castets, A., Steinacker, J., \& Loinard, L. 2002, A\&A 389, L6

Bacmann, A., Lefloch, B., Ceccarelli, C., Steinacker, J., Castets, A., \& Loinard, L. 2003, Ap. J. 585, L55

Boogert, A.C.A., Hogerheijde, M.R., Ceccarelli, C., Tielens, A.G.G.M., van Dishoeck, E.F., Blake, G.A., Latter, W.B., \& Motte, F. 2002, Ap. J. 570, 708

Bottinelli, S., Ceccarelli, C., Lefloch, B., Williams, J.P., Castets, A., Caux, E., Cazaux, S., Maret, S., Parise, B., \& Tielens, A.G.G.M. 2004a, Ap. J. 615, 354

Bottinelli, S., Ceccarelli, C., Neri, R., \& Williams, J.P. 2005a, Ap. J., in preparation

Bottinelli, S., Ceccarelli, C., Neri, R., Williams, J.P., Caux, E., Cazaux, S., Lefloch, B., Maret, S., \& Tielens, A.G.G.M. 2004b, Ap. J. 617, L69

Bottinelli, S., Ceccarelli, C., Williams, J.P., \& Lefloch, B. 2005b, Ap. J., submitted

Caselli, P., van der Tak, F.F.S., Ceccarelli, C., \& Bacmann, A. 2003, A\&\&A 403, L37

Cazaux, S., Tielens, A.G.G.M., Ceccarelli, C., Castets, A., Wakelam, V., Caux, E., Parise, B., \& Teyssier, D. 2003, Ap. J. 593, L51

Ceccarelli, C. 2004a, in The Dense Interstellar Medium in Galaxies, ed. S. Pfalzner et al. (Berlin: Springer Verlag), 473

Ceccarelli, C. 2004b, in Star Formation in the Interstellar Medium, ed. D. Johnstone et al. (ASP Conference Series), 323, 195 
Ceccarelli, C., Castets, A., Caux, E., Hollenbach, D., Loinard, L., Molinari, S., \& Tielens, A.G.G.M. 2000a, A\&A 355, 1129

Ceccarelli, C., Castets, A., Loinard, L., Caux, E., \& Tielens, A.G.G.M. 1998, A\&\&A 338, L43

Ceccarelli, C., Caux, E., Loinard, L., Castets, A., Tielens, A.G.G.M., Molinari, S., Liseau, R., Saraceno, P., Smith, H., \& White, G. 1999, A\&A 342, L21

Ceccarelli, C., \& Dominik, C. 2005, A\&A 440, 583

Ceccarelli, C., Hollenbach, D.J., \& Tielens, A.G.G.M. 1996, Ap. J. 471, 400

Ceccarelli, C., Loinard, L., Castets, A., Tielens, A.G.G.M., \& Caux, E. 2000b, A\& $A$ 357, L9

Ceccarelli, C., Loinard, L., Castets, A., Tielens, A.G.G.M., Caux, E., Lefloch, B., \& Vastel, C. 2001, A\&BA 372, 998

Chandler, C.J., Brogan, C.L., Shirley, Y.L., \& Loinard, L. 2005, Ap. J. 632, 371

Charnley, S.B. 1997, Ap. J. 481, 396

Charnley, S.B., Tielens, A.G.G.M., \& Rodgers, S.D. 1997, Ap. J. 482, L203

Chen, H., Myers, P.C., Ladd, E.F., \& Wood, D.O.S. 1995, Ap. J. 445, 377

Dartois, E., Thi, W.-F., Geballe, T.R., Deboffle, D., d'Hendecourt, L., \& van Dishoeck, E. 2003, A\&A 399, 1009

Doty, S.D., Schoier, F.L., \& van Dishoeck, E.F. 2004, A\&A 418, 1021

Flower, D.R., Pineau des Forêts, G., \& Walmsley, C.M. 2004, A\&A 427, 887

Froebrich, D. 2005, Ap. J. Suppl. 156, 169

Hatchell, J., Roberts, H., \& Millar, T.J. 1999, A\&SA 346, 227

Jorgensen, J.K., Bourke, T.L., Myers, P.C., Schöier, F.L., van Dishoeck, E.F., \& Wilner, D.J. 2005a, Ap. J. 632, 973

Jorgensen, J.K., Schöier, F.L., \& van Dishoeck, E.F. 2002, A\&SA 389, 908

- 2005b, A\& A 437, 501

Kuan, Y.-J., Huang, H.-C., Charnley, S.B., Hirano, N., Takakuwa, S., Wilner, D.J., Liu, S.-Y., Ohashi, N., Bourke, T.L., Qi, C., \& Zhang, Q. 2004, Ap. J. 616, L27

Kurtz, S., Cesaroni, R., Churchwell, E., Hofner, P., \& Walmsley, C.M. 2000, Protostars and Planets IV, 299

Lee, J.-E., Bergin, E.A., \& Evans, N.J. 2004, Ap. J. 617, 360

Lis, D.C., Roueff, E., Gerin, M., Phillips, T.G., Coudert, L.H., van der Tak, F.F.S., \& Schilke, P. 2002, Ap. J. 571, L55

Loinard, L., Castets, A., Ceccarelli, C., Caux, E., \& Tielens, A.G.G.M. 2001, Ap. J. 552, L163

Loinard, L., Castets, A., Ceccarelli, C., Lefloch, B., Benayoun, J.-J., Caux, E., Vastel, C., Dartois, E., \& Tielens, A.G.G.M. 2002, Planet. Space Sci. 50, 1205

Loinard, L., Castets, A., Ceccarelli, C., Tielens, A.G.G.M., Faure, A., Caux, E., \& Duvert, G. 2000, A\& $A 359,1169$

Maret, S., Ceccarelli, C., Caux, E., Tielens, A.G.G.M., \& Castets, A. 2002, A $8 A$ 395, 573

Maret, S., Ceccarelli, C., Caux, E., Tielens, A.G.G.M., Jörgensen, J.K., van Dishoeck, E., Bacmann, A., Castets, A., Lefloch, B., Loinard, L., Parise, B., \& Schöier, F.L. 2004, A $\& A$ 416,577

Maret, S., Ceccarelli, C., Tielens, A.G.G.M., Caux, E., Lefloch, B., Faure, A., Castets, A., \& Flower, D.R. 2005, $A \& A$, in press

Osamura, Y., Roberts, H., \& Herbst, E. 2004, A\&A 421, 1101

Parise, B., Castets, A., Herbst, E., Caux, E., Ceccarelli, C., Mukhopadhyay, I., \& Tielens, A.G.G.M. 2004, $A \& A 416,159$

Parise, B., Caux, E., Castets, A., Ceccarelli, C., Loinard, L., Tielens, A.G.G.M., Bacmann, A., Cazaux, S., Comito, C., Helmich, F., Kahane, C., Schilke, P., van Dishoeck, E., Wakelam, V., \& Walters, A. 2005, A\&A 431, 547

Parise, B., Ceccarelli, C., Tielens, A.G.G.M., Herbst, E., Lefloch, B., Caux, E., Castets, A., Mukhopadhyay, I., Pagani, L., \& Loinard, L. 2002, A\&A 393, L49

Parise, B., Simon, T., , Caux, E., Dartois, E., Ceccarelli, C., Rayner, J., \& Tielens, A.G.G.M. 2003, A\&BA, 410, 897

Roberts, H., Herbst, E., \& Millar, T.J. 2003, Ap. J. 591, L41

Roberts, H., \& Millar, T.J. 2000, A\& A 364, 780

Rodgers, S.D., \& Charnley, S.B. 2003, Ap. J. 585, 355 
Roueff, E., Tiné, S., Coudert, L.H., Pineau des Forêts, G., Falgarone, E., \& Gerin, M. 2000, A\&A 354, L63

Schöier, F.L., Jorgensen, J.K., van Dishoeck, E.F., \& Blake, G.A. 2002, A\&\&A 390, 1001

Shu, F.H. 1977, Ap. J. 214, 488

Stantcheva, T., \& Herbst, E. 2003, MNRAS 340, 983

Stauber, P., Doty, S.D., van Dishoeck, E.F., \& Benz, A.O. 2005, A $\mathscr{J} A$ 440, 949

Stauber, P., Doty, S.D., van Dishoeck, E.F., Jorgensen, J.K., \& Benz, A.O. 2004, A $\mho A$ 425, 577

Tielens, A.G.G.M. 1983, A\&A 119, 177

Tielens, A.G.G.M., \& Hagen, W. 1982, A\&A 114, 245

Turner, B.E. 1990, Ap. J. 362, L29

van der Tak, F.F.S., Schilke, P., Müller, H.S.P., Lis, D.C., Phillips, T.G., Gerin, M., \& Roueff, E. 2002, A\&SA 388, L53

Vastel, C., Phillips, T.G., Ceccarelli, C., \& Pearson, J. 2003, Ap. J. 593, L97

Vastel, C., Phillips, T.G., \& Yoshida, H. 2004, Ap. J. 606, L127

Wakelam, V., Caselli, P., Ceccarelli, C., Herbst, E., \& Castets, A. 2004a, A $\& A$ 422, 159

Wakelam, V., Castets, A., Ceccarelli, C., Lefloch, B., Caux, E., \& Pagani, L. 2004b, A $8 A$ 413, 609

Wakelam, V., Ceccarelli, C., Castets, A., Lefloch, B., Loinard, L., Faure, A., Schneider, N., \& Benayoun, J.-J. 2005, A\&A 437, 149

Walmsley, M., Flower, D., \& des Forets, G.P. 2004, A\&A 418, 1035

\section{Discussion}

RAWLings: Concerning the so-called "hot corinos": these objects are barely resolvable, so what evidence is there that they are the low-mass analogues of hot cores, rather than say - outflow interfaces?

Wootten: In the two binary objects, only one component shows hot corino aspects. In both, that same component is the only one powering a flow, seen on scales as small as $1 / 3 \mathrm{AU}$ in 16293 in $\mathrm{H}_{2} \mathrm{O}$ maser emission. Since we know there are active flow-driven chemistries occurring in the two hot-corino sources, is it is perhaps too early to rule out the contributions of outflows to enhancement of emissions from more complex molecules? To discern the roles played by outflow and by protostellar heating, we need more sensitivity and more resolution, such as will be provided by ALMA.

Ceccarelli: Although it is possible, in principle, that the observed jumps in the abundances of typical hot core molecules are caused by the interaction between the outflow and the envelope, several reasons are in favor of the dust sublimation region (hot corino) hypothesis: (1) This is a very simple and natural explanation, which is based on the basic prediction that the temperature of the envelope increases towards the center, coupled with the SED+SCUBA map observations fits. (2) The high-resolution maps of the complex organic molecules (Kuan et al. 2004; Bottinelli et al. 2004) do not show any hint of the outflow interaction, but agree with the point (1) predictions.

GLASSGOLD: If IRAS 16293 is not a "freak", why is the size of its hot inner region so much larger than other Class 0 sources?

CecCarelli: The size of the hot inner region depends on the luminosity and density. IRAS 16293 is relatively bright $\left(\sim 15 \mathrm{~L}_{\odot}\right)$ and with a relatively low-mass envelope with respect to the other studied Class 0 sources. Whether this is because IRAS 16293 is younger or less massive than the other sources is not clear. The remarkable fact is that it is chemically representative of the other sources. This says to us that the initial conditions 
are, after all, not too different in Class 0 sources, based on both the deuteration degree and the measure of complex organic molecules.

GUÉLIN: You have compared in a table the fractional abundances of molecules in the hot and cold components of several "corinos". The hot-core component is, however, hardly or unresolved even with interferometers like the BdBI or SMA. How can you then compare the beam-averaged abundances in unresolved sources, which lie at different distances and presumably have different sizes?

CecCARELli: The abundances have been derived by comparing the observations with the predictions of a model which takes into account the density and temperatures profiles of each studied source. The profiles have been derived from the SED and SCUBA maps of each source - based on this structure the model predicts the sizes where the dust temperature exceeds $100 \mathrm{~K}$, the sublimation temperature of the water ices (in first approximation). Therefore, the derived abundances, although they are model dependent, are not derived by "simple" beam-averaged column densities.

NEUfELD: If I understand you correctly, you have assumed a value of $100 \mathrm{~K}$ for the temperature at which the "hot-core chemistry", as you call it, turns on. This reflects a theoretical presumption about what these regions are. Is it possible, in cases where you have large numbers of observed transitions, to treat the "turn-on" temperature as a free parameter that will be constrained by the observations?

CecCARelli: Indeed, we have evidence that icy mantles start to sublimate at a lower temperature in IRAS 16293-2422, based on the $\mathrm{H}_{2} \mathrm{CO}$ observations (see Ceccarelli et al. 2001), because of the large number of observed transitions. As regards the organic complex molecules, sign spots of the "hot core chemistry", the best way to constrain the sublimation mantle temperature is so far the interferometric observations obtained toward IRAS 16293-2422 (Kuan et al. 2004; Bottinelli et al. 2004). But, in principle, yes, a multi-frequency study with a large number of transition can also constrain the "turn-on" temperature, as in the case of $\mathrm{H}_{2} \mathrm{CO}$. 


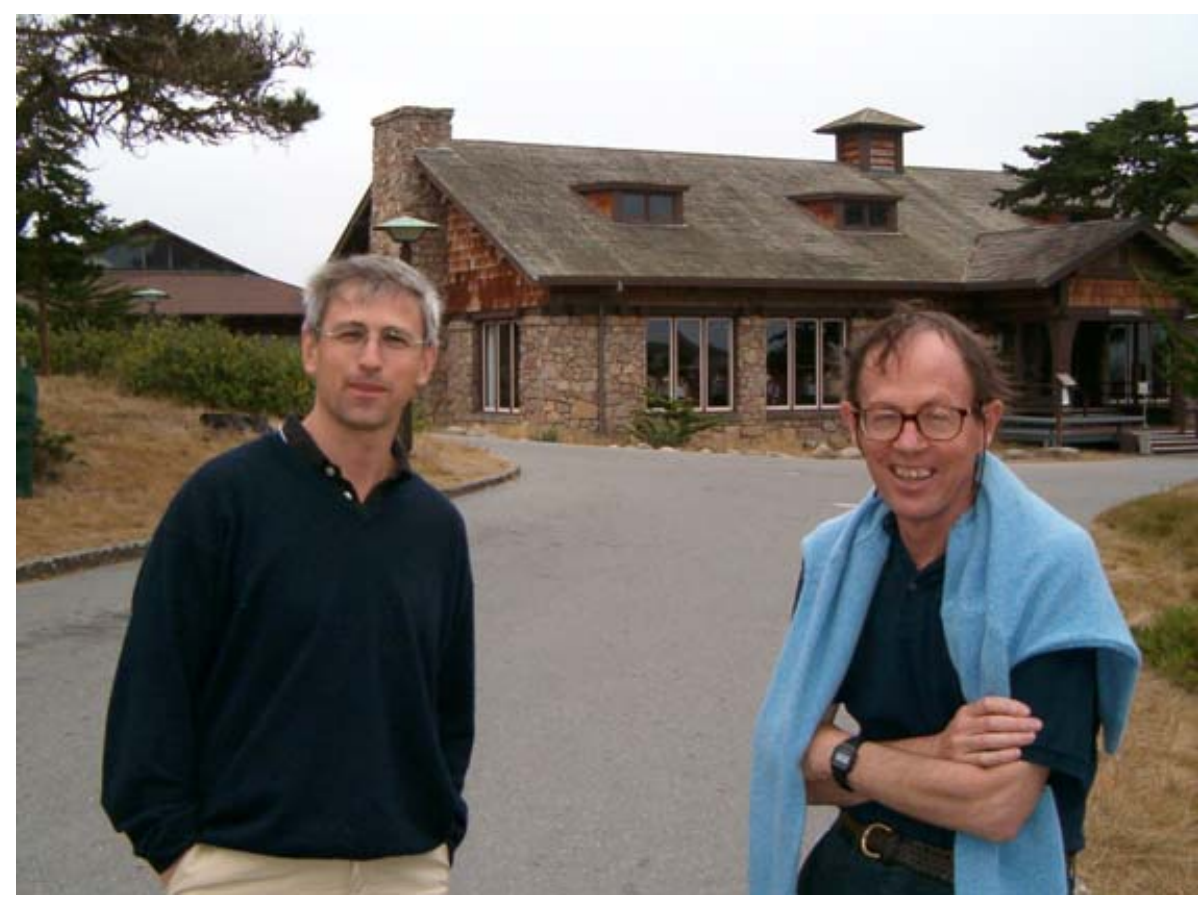

Photo: E. Herbst 